\title{
Squeeze Film Effect for the Design of an Ultrasonic Tactile Plate
}

\author{
Mélisande Biet, Frédéric Giraud, Member, IEEE, and Betty Lemaire-Semail, Member, IEEE
}

\begin{abstract}
Most tactile displays currently built rely on pin-based arrays. However, this kind of tactile device is not always appropriate when we need to give the illusion of finely textured surfaces. In this paper, we describe the squeeze film effect between a plate and a finger, and we use this effect to design an ultrasonic tactile plate. The plate is actuated by piezoelectric ceramics. Ultrasonic vibrations are thus produced and are capable of generating the squeeze film effect. This enables us to simulate variable friction on the surface of the plate. In order to identify the squeeze film phenomenon, this study considers the case where a finger, with a planar bottom surface and with epidermal ridges, is placed on a rapidly vibrating plate. The overpressure is calculated and the result enables us to assess the relative coefficient of friction as a function of the vibration amplitude of the plate. Based on this principle, and using both analytic and FE method studies, and given ergonomic and stimulation (squeeze film) requirements, we show that it is possible to design a tactile plate which is capable of giving programmable tactile sensations. We conclude by comparing the results obtained from our simulations with experimental results.
\end{abstract}

\section{INTRODUCTION}

$\mathrm{W}$ E experience everyday shapes and textures through different degrees of skin deformation, and changes in thermal boundary conditions, as well as localized and distributed vibrations at the skin surface. These different interactions with various surfaces allow us to explore and feel our environment. However, human-computer interaction technology has rarely relied on touch to close the communication loop used to handle the information transfer from the computer to the user. In fact, despite the huge number of devices produced with various manufacturing and actuation technologies [1], most of the tactile displays built to this day fail to efficiently deform the skin and be practical at the same time. For instance, in order to create discrete representations of a texture or a small shape, pin based arrays are commonly used [2]. But, with regard to ecommerce, for example, where it would be useful to be able to feel a material via the internet, this kind of tactile device is not appropriate to simulate finely textured surfaces because miniaturization and technical integration problems are still unresolved. Miniaturization will inevitably reach its limits and pin array technology may impose technological and cost constraints.

Manuscript received February 19, 2007; accepted July 31, 2007. This work was carried out within the framework of the INRIA Alcove project and is supported by the IRCICA (Institut de Recherche sur les Composants logiciels et matériels pour l'Information et la Communication Avancée) and The European Commission (FEDER).

The authors are with the University of Lille, L2EP, Villeneuve D'Ascq, France (e-mail: melisande.biet@polytech-lille.fr).

Digital Object Identifier 10.1109/TUFFC.2007.596
The problem has been approached in various ways. For example, Hayward et al. have suggested creating tactile sensations by deforming the skin laterally, with negligible normal deflection, enabling miniaturization [3]. Another possibility is the use of surface acoustic waves [4], [5] or ultrasonic vibrations [6] that modify the sliding interaction between a surface and a finger.

Following the same idea, in order to simulate finely textured surfaces, we use the squeeze film effect principle. It has been shown that a beam excited by ultrasonic vibration can give rise to smooth or unsmooth feelings according to the amplitude of the vibrations [6]. Moreover, the feeling of roughness can be generated by imposing lowfrequency periodic signals over the ultrasonic vibration. We have studied this phenomenon on the stator of an ultrasonic wave motor by controlling the space period variation of the waves [7]. As a result, the smooth feeling is also modulated: We obtain a virtual texture which is alternately smooth and unsmooth. This feeling is interpreted as ridges and grooves by users. This technology is especially relevant for obtaining more complex spatial properties with a lower number of actuators. Nevertheless, before designing a tactile plate able to simulate these kinds of sensations, it is necessary to better identify what happens at the contact area between a vibrating surface and a finger. In this study, it was deemed relevant to take into account the fingerprint undulations in order to calculate the overpressure (a transient air pressure that is greater than the surrounding atmospheric pressure) created between the two surfaces. This is carried out in the second part of the paper. Moreover, this overpressure also enables us to find the relative coefficient of friction as a function of the vibration amplitude of the acoustic wave. We thus obtain one of the criteria necessary for the design of the tactile plate.

This plate (Fig. 1) can bend by means of PZT piezo-ceramics glued onto one of the two surfaces. The monomorph constructed in this manner is the subject of analytical and numerical studies (Section III). Design of the tactile plate based on theoretical analysis and experimental evaluation is then discussed in the fourth part.

\section{Squeeze Film Effect Between a Vibrating Surface AND A Finger}

The goal of this section is to calculate the overpressure caused by the squeeze effect. It will then be possible to find the conditions under which this force leads to a variation of the friction coefficient of the surface. In this section, the ge- 


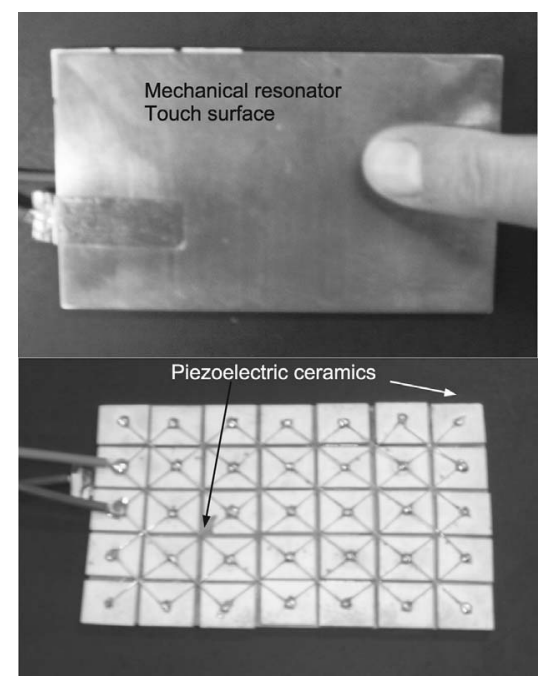

Fig. 1. Complete view of the actuator. Below: the piezo-ceramics matrix; above: the touch surface.

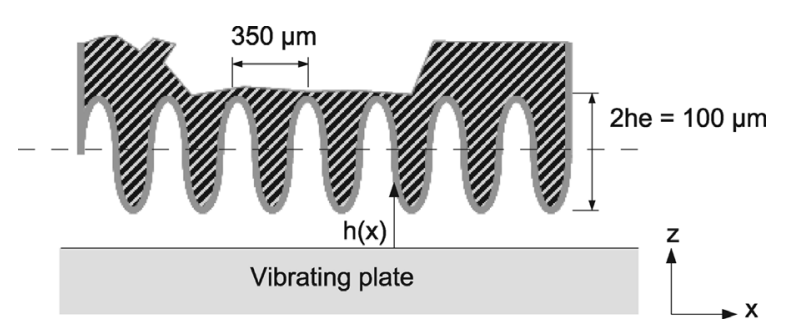

Fig. 2. Approximate profile of a fingertip when the epidermal ridges are taken into account.

ometric properties of the fingerprints (or epidermal ridges) are taken into account. In fact, we cannot suppose that the undulations of the fingerprints are negligible relative to the roughness of the vibrating plate (a few micrometers) since the height $\left(2 h_{e}\right)$ and pitch $(L)$ of the epidermal ridge are around $100 \mu \mathrm{m}$ and $350 \mu \mathrm{m}$, respectively [8] (Fig. 2).

\section{A. Squeeze Film Model}

In this subsection, we describe the squeeze effect [9]. It is thus necessary to consider the gas film created between the fingertip and a planar vibrating object. We rely on a study developed in [10], but take into account the undulations of the fingerprints. We regard the tip of the finger as an undulated surface while the planar plate is assumed to oscillate sinusoidally in a vertical direction (Fig. 2).

The film thickness, $h$, is the sum of the gap created when the skin of the finger is in no way able to follow the ultrasonic vibration of the plate. This $h$ thus equals the amplitude of oscillation, $h_{\mathrm{vib}}$, plus the surface unevenness (roughness), $h_{r}$. At last, $h$ has also to take into account the amplitude of the undulation of the fingerprint, $h_{e}$. Therefore, the thickness of the film is given by [6]

$$
\begin{aligned}
& h(x, t)= \\
& \quad h_{r}+h_{\mathrm{vib}}\left[1+\cos \left(\omega_{0} t\right)\right]+h_{e}\left[1+\cos \left(\frac{2 \pi}{L} x\right)\right],
\end{aligned}
$$

where the vibrating frequency of the plate is given by $\omega_{0}$. If we normalize $h$, the normalized airgap can be written with nondimensional parameters as

$$
H=1+\epsilon \cos (T)+\delta \cos (k X)
$$

When $h_{0}=h_{\mathrm{vib}}+h_{r}$, the nondimensional parameters are

$$
\begin{aligned}
H & =\frac{h}{h_{0}+h_{e}}, \quad X=\frac{x}{l_{0}}, \quad T=\omega_{0} t, \\
\epsilon & =\frac{h_{\mathrm{vib}}}{h_{0}+h_{e}}, \quad \delta=\frac{h_{e}}{h_{0}+h_{e}}, \quad k=\frac{2 \pi l_{0}}{L},
\end{aligned}
$$

where the length in contact with the fingertip is $l_{0}$.

We make the following assumptions [10]: 1) The fluid behavior is governed by laminar viscous flow, 2) the fluid is a compressible perfect gas, 3 ) the inertia effect of the flow is negligible, and 4) the relative lateral motion is equal to zero. Under those conditions, the one-dimensional Navierstock equation is obtained. This equation, when used in association with continuity and ideal gas equations, allows us to find the governing Reynolds equation in the nondimensional form:

$$
\nabla\left(H^{3} P^{1 / n} \cdot \nabla(P)\right)=\sigma \frac{\partial\left(P^{1 / n} H\right)}{\partial T},
$$

where $n$ is a polytropic constant and

$$
P=\frac{p}{p_{0}}, \quad \sigma=\frac{12 \eta \omega_{0} l_{0}}{p_{0}\left(h_{0}+h_{e}\right)^{2}}
$$

where $p, p_{0}$, and $\eta$ represent the pressure in the gap, the surrounding gas pressure, and the dynamic viscosity of the fluid, respectively. The squeeze number is given by $\sigma$, which represents a measurement of the fluid compressibility in the gap. At low squeeze numbers, the fluid is nearly incompressible, while at high squeeze numbers, the fluid is trapped in the gap and acts like a spring.

We further assume that the squeezed film is isothermal $(n=1)$. This assumption is reasonable since the gas film is very thin and of low heat capacity when compared with that of the vibrating surfaces. Then, to simplify the time derivation term, we substitute $P H$ with $\Psi$. For steadystate conditions, integration with respect to one period yields [10]

$$
\nabla\left[\frac{1}{2} \bar{H} \nabla\left(\Psi_{\infty}^{2}\right)-\Psi_{\infty}^{2} \nabla(\bar{H})\right]=0
$$

where $\Psi_{\infty}$ denotes the $\Psi$ inside the airgap when $\sigma \rightarrow \infty$ and $\bar{H}$ is the mean normalized film thickness given by

$$
\bar{H}=1+\delta \cos (k X) .
$$

It should be noted that the analytical solution is accurate as long as $\sigma$ is assumed to be a very large value. On the contrary, a low value of $\sigma$ would imply a much more complex solution. 


\section{B. Approximate Analytical Solution}

Eq. (5) gives us the relationship between the normalized film thickness and the normalized pressure for a fluid behavior close to a spring. The solution of (5) is given by (Appendix A) [10]:

$$
\Psi_{\infty}^{2}=K_{1}[1+\delta \cos (k X)]^{2},
$$

where $K_{1}$ is a constant. The boundary conditions with respect to $\Psi_{\infty}^{2}(X)$, represented by $\Psi_{\infty B}$, are

$$
\Psi_{\infty B}^{2}\left(-\frac{1}{2}\right)=\Psi_{\infty B}^{2}\left(\frac{1}{2}\right)=K_{1}\left[1+\delta \cos \left(\frac{k}{2}\right)\right]^{2},
$$

where $1 / 2$ is the normalized value of $\left(l_{0} / 2\right)$. Replacing $K_{1}$ by its expression in (7), we obtain

$$
\Psi_{\infty}=\Psi_{\infty B}\left(\frac{1}{2}\right) \frac{|1+\delta \cos (k X)|}{\left|1+\delta \cos \left(\frac{k}{2}\right)\right|} .
$$

Furthermore, in order to find $\Psi_{\infty B}$, we will focus our attention on the boundary region. Considering that $p_{0}$ is constant in time and that $\Psi_{\infty B}$ equals $\Psi_{\infty}$ when the boundary region meets the interior of the airgap, we obtain [10]

$$
\begin{aligned}
\Psi_{\infty B}^{2}\left(\frac{1}{2}\right) & =p_{0}^{2} \frac{\int_{T}^{T+2 \pi} H_{B}^{3} d T}{\int_{T}^{T+2 \pi} H_{B} d T} \\
& =p_{0}^{2} \frac{\int_{T}^{T+2 \pi}\left[1+\epsilon \cos (T)+\delta \cos \left(\frac{k}{2}\right)\right]^{3} d T}{\int_{T}^{T+2 \pi}\left[1+\epsilon \cos (T)+\delta \cos \left(\frac{k}{2}\right)\right] d T} \\
& =p_{0}^{2}\left[1+\delta \cos \left(\frac{k}{2}\right)\right]\left[1+\frac{3}{2} \frac{\epsilon^{2}}{\left[+\delta \cos \left(\frac{k}{2}\right)\right]^{2}}\right] .
\end{aligned}
$$

Replacing $\Psi_{\infty B}$ by its expression in (9), we obtain:

$$
\begin{aligned}
P_{\infty} & =\frac{\Psi_{\infty}}{H} \\
& =p_{0} \frac{(1+\delta \cos (k X)) \sqrt{\left(1+\delta \cos \left(\frac{k}{2}\right)\right)^{2}+\frac{3}{2} \epsilon^{2}}}{\left(1+\delta \cos \left(\frac{k}{2}\right)\right)(1+\epsilon \cos (T)+\delta \cos (k X))} .
\end{aligned}
$$

We should remember that $P_{\infty}$ is the normalized pressure inside the airgap for an infinite value of the squeeze number $\sigma$. With $\Omega$ as a variable position on the airgap surface, the nondimensional average squeeze force $F_{s}$ per unit length at steady state can be expressed by

$$
\begin{aligned}
F_{s} & =\iint_{\Omega}\left(P_{\infty}-1\right) d \Omega \\
& =\frac{1}{2 \Pi} \int_{0}^{2 \pi}\left(\int_{-\frac{1}{2}}^{\frac{1}{2}}\left(P_{\infty}-1\right) d X\right) d T .
\end{aligned}
$$

The mean squeeze pressure is thus given by

$$
\overline{P_{\infty}}=p_{0} \frac{(1+\delta \cos (k X)) \sqrt{\left(1+\delta \cos \left(\frac{k}{2}\right)\right)^{2}+\frac{3}{2} \epsilon^{2}}}{\left(1+\delta \cos \left(\frac{k}{2}\right)\right) \sqrt{(1+\delta \cos (k X))^{2}-\epsilon^{2}}} .
$$

TABLE I

List of PARAmeters.

\begin{tabular}{lll}
\hline Amplitude of the fingerprint & $h_{e}$ & $50[\mu \mathrm{m}]$ \\
Period of the fingerprint & $L$ & $350[\mu \mathrm{m}]$ \\
Average roughness of the plate & $h_{r}$ & $0.4-0.8-1.2-1.6[\mu \mathrm{m}]$ \\
Length in contact & $l_{0}$ & $1[\mathrm{~cm}]$ \\
Force applied by the fingertip & $F_{f}$ & $0.3 \rightarrow 0.7[\mathrm{~N}]$ \\
Dynamic viscosity of air $\left(\right.$ at $\left.20^{\circ} \mathrm{C}\right)$ & $\eta$ & $1.85 .10^{-5}[\mathrm{~Pa} . \mathrm{s}]$ \\
Atmospheric pressure & $p_{0}$ & $0.1[\mathrm{MPa}]$ \\
\hline
\end{tabular}

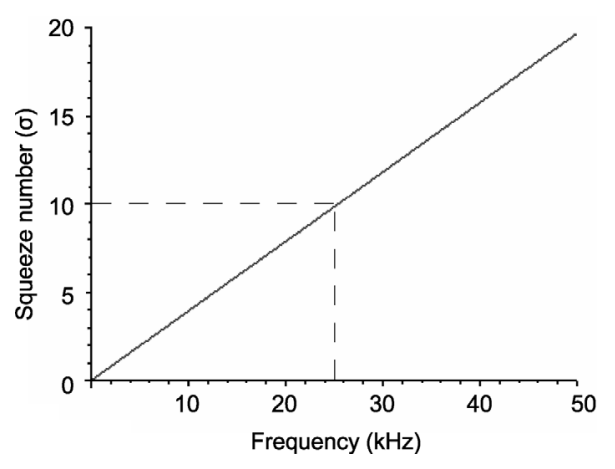

Fig. 3. Squeeze number as a function of the vibration frequency of the plate for $h_{r}=1.6 \mu \mathrm{m}$ and $h_{\mathrm{vib}}=3 \mu \mathrm{m}$.

\section{Results of the Model}

First, let us note that in order to predetermine analytically the overpressure on the fingertip, the following condition must be satisfied: $\sigma \rightarrow \infty$. However, we assume in practice that the squeeze force (12) depends almost entirely on the amplitude of vibration when the squeeze number is larger than 10. Therefore, we calculate the squeeze number $\sigma$, as shown in (4), with the parameters of Table I, as a function of the frequency of the oscillation for a given amplitude $h_{\mathrm{vib}}$.

Considering the most unfavorable case, i.e., the smallest slope of the straight line representing $\sigma=f\left(\omega_{0}\right)$ (a surface state, $h_{r}$, of $1.6 \mu \mathrm{m}$ and a vibration amplitude, $h_{\mathrm{vib}}$, of $3 \mu \mathrm{m}$ ), the results (Fig. 3) enable us to determine the first criterion of the frequency $(f>25 \mathrm{kHz})$, which in turn allows us to use (5).

Using the parameters shown in Table I, we can compute the pressure profile along the airgap using (11). According to the previous results, the frequency of the oscillation is set to $40 \mathrm{kHz}$ in order to exceed $25 \mathrm{kHz}$.

On the upper part of Fig. 4, the finger, which is in contact with the plate, is modeled as a sinusoid with an amplitude of $50 \mu \mathrm{m}$ in order to take the fingerprints into account. The interval from zero depicts the mean roughness plus the vibration amplitude of the vibrating plate itself. The curve below shows the evolution of the average temporal pressure as a function of the position on the $x$ axis. In Fig. 4, pressure peaks are localized where the skin of the finger is as close as possible to the vibrating plate, i.e., at the fingerprint ridges, whereas at the fingerprint grooves, pressure is similar to the atmospheric pressure. 

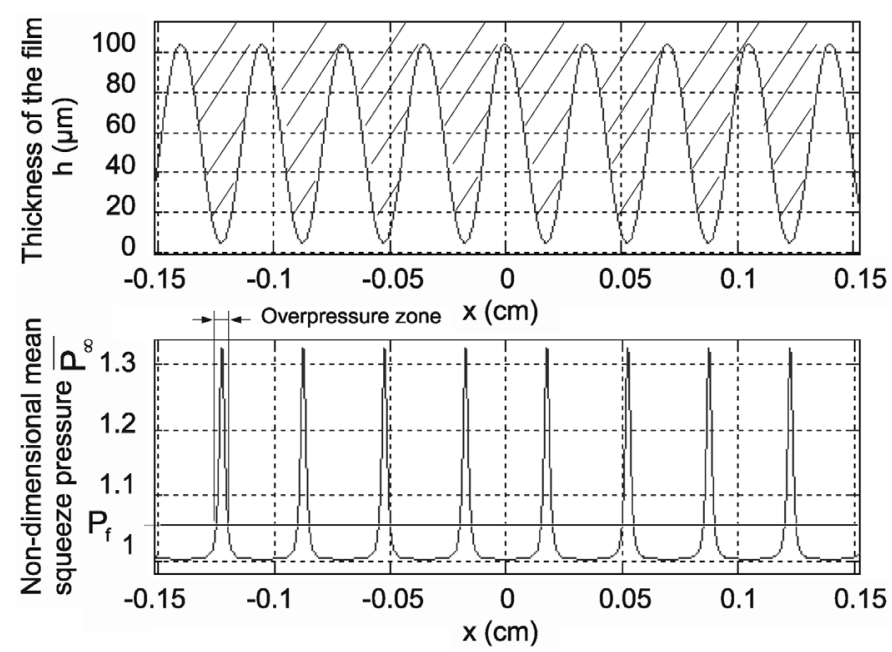

Fig. 4. Thickness of the film at a given time and spatial profile of the mean time pressure under the finger $\left(h_{r}=1.6 \mu \mathrm{m}, h_{\mathrm{vib}}=3 \mu \mathrm{m}\right)$.

The pressure $P_{f}$ is the mean finger pressure that is used during an exploration task:

$$
P_{f}=F_{f} / l_{0}^{2},
$$

where $F_{f}$ denotes the normal contact force applied by a person exploring the surface. For its value, we choose $0.5 \mathrm{~N}$ as a mean normal contact force, in accordance with [6], [11].

We can see from Fig. 4 that there are some zones of the fingerprint where the squeeze pressure is superior to the finger pressure. Those zones are not in contact with the vibrating plate.

Moreover, when the squeeze force occurs, we can express the relative coefficient of friction, $\left(\mu^{\prime} / \mu\right)$, as shown in

$$
\frac{\mu^{\prime}}{\mu}=1-\frac{\left(P_{\infty}-1\right)}{P_{f}}
$$

Physically, and following the assumptions previously defined, this equation means a weakening of the friction coefficient for a given surface when the squeeze force occurs.

To illustrate the friction coefficient variations, we can compute $\left(\mu^{\prime} / \mu\right)$ for four cases of surface roughness (Table I). The results are given in Fig. 5 .

We can easily calculate the relative friction coefficient, taking the fingerprints into account. However, our results are more pessimistic than those of Watanabe [6]. In fact, for an average roughness of a few micrometers (which is realistic from a technological point of view), a vibration amplitude of the plate higher than $4 \mu \mathrm{m}$ would be necessary to reach a zero relative friction coefficient instead of only $1 \mu \mathrm{m}$, as found in [6]. It would seem that this is due to the consideration of the fingerprint in our study. Hence, taking into account the lubrication effect (squeeze film effect), we can succeed in changing the sensation by exploiting the friction coefficient between the finger and the plate, an approach which was verified quantitatively in [12].

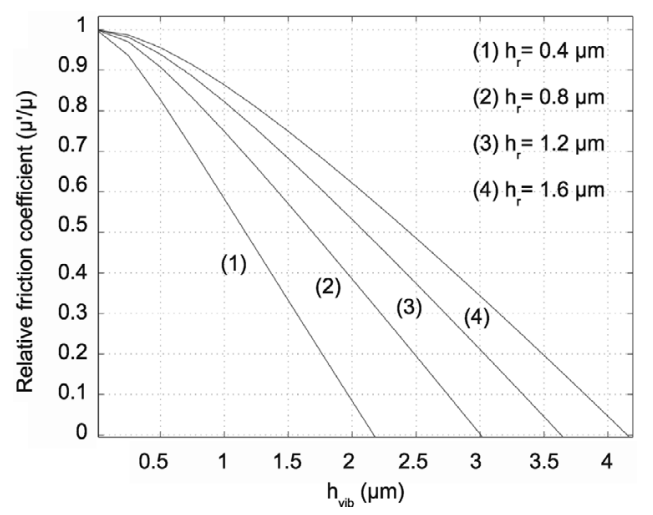

Fig. 5. The relative coefficient of friction as a function of the amplitude of vibration for $F_{f}=0.5 \mathrm{~N}$ and for the four values of $h_{r}$ (Table I).

We should note that the relative coefficient of friction is strongly dependent on the force applied by the user $\left(F_{f}\right)$; thus the results of Fig. 5 should be considered with care.

\section{Guidelines for the Design}

Using the results of Fig. 5, it is possible to foresee the vibration amplitude required to levitate the fingertip, i.e., the amplitude for which the relative coefficient of friction is zero. Nevertheless, the goal of our tactile device is not to induce finger levitation. Our sole aim is to decrease the friction coefficient between the surface and the finger in a significant way in order to make the user feel the difference of perception. This analysis thus gives us the information necessary for the design of the plate. For instance, a vibration amplitude of $1.5 \mu \mathrm{m}$ with a carefully prepared surface $\left(h_{r}=0.6 \mu \mathrm{m}\right)$ would be enough for the user to feel the difference of perception (half of the relative friction coefficient).

\section{Design of the Tactile Plate}

The results that we have obtained thus far allow us to determine the range of the vibration amplitude we must impose on the plate in order to obtain the squeeze film effect. Our goal in this section is to find the dimensions of the composite plate (monomorph) in order to fulfill ergonomic, amplitude, and frequency requirements. To achieve this goal, piezoceramics were glued to the plate, which created ultrasonic vibrations. We thus generated an ultrasonic standing wave in the plate. It should be noted that the motion of the surface points are no longer uniform in that case. Nevertheless, we make the assumption that the overpressure will still exist. The results will be checked experimentally in the final section of this paper.

With regard to our analysis of the analytical deflection, the simple structure considered is the half-wavelength portion of a heterogeneous beam with a rectangular section. This analysis enables us to determine the deflection of the beam as a function of geometrical parameters. The reso- 


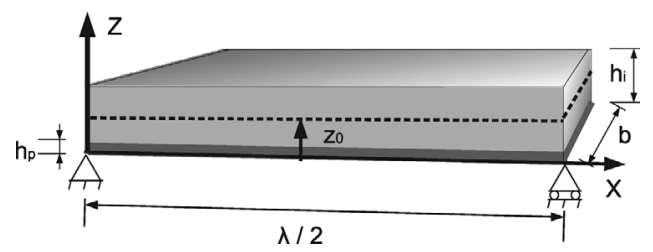

Fig. 6. A half-wavelength section of the monomorph is modeled as a simply supported beam.

nance frequency is then calculated as a function of geometrical parameters. Our results enable us to delimit the numerical study. The numerical study is carried out using finite element computation software and allows us to justify some of the assumptions of the model.

\section{A. Design Requirements: An Ergonomic Workspace}

The tactile device must fulfill mechanical requirements, but it also has to be compatible with the user's movement. Therefore, defining an ergonomic workspace is a key issue for designing such devices. According to [13], when the user is trying to recognize a finely textured surface, it is important to "allow the freedom of active exploration" on a surface perceived as infinite by the user. Klatzky and Lederman first observed that, during an exploration task which they carried out as part of their study, "the fingers quickly rub back and forth along a small, homogeneous area of the surface," and that "interior surfaces are explored rather than edges" [14]. The exploration surface is thus bounded, and Martinot in [15] measures its average dimension: To achieve this, the author collects the position and normal force exerted by the fingertip while volunteers actively explore finely textured surfaces. He shows that the user's movement is in average limited to a $45 \mathrm{~mm} \times 57 \mathrm{~mm}$ rectangle. This means that in the absence of vision, a workspace of this dimension will give the impression of a very large surface.

\section{B. Analytical Study}

The analytical study is carried out in two steps. The first step begins with an analysis of the static deflection of the plate caused by piezoelectric excitation. We examine a half-wavelength portion of the plate, $\lambda / 2$, modeled as a simply supported beam, as illustrated in Fig. 6. After determining the static deflection of the plate, the deflection magnitude at resonance can be calculated by multiplying the static value by a dynamic amplification factor. The second step involves calculating the resonant frequency as a function of the half wavelength for a given width.

1. Determination of the Static Deflection: The considered monomorph (Fig. 6) consists of a layer of piezoelectric ceramics, thickness $h_{p}$, and of a layer of passive material (copper beryllium, for example), which we call the mechanical resonator, thickness $h_{i}$. Ceramic and substrate are stuck together. The working assumption retained is

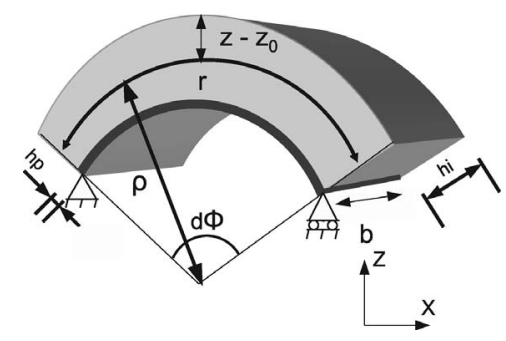

Fig. 7. Half-wavelength beam which bends by contracting the piezoceramics.

that this assembly is perfect, which amounts to considering that the thickness of adhesive is zero and that the strains are continuous on the substrate-piezoelectric ceramic interface. The beam's length is $\lambda / 2$ and its width is $b$.

First, the displacement vector in the cartesian frame is described using the Bernoulli-Euler theory [16]:

$$
U=\left(\begin{array}{c}
u \\
v \\
w
\end{array}\right)=\left(\begin{array}{c}
\left(z_{0}-z\right) \frac{\delta w}{\delta x} \\
\left(z_{0}-z\right) \frac{\delta w}{\delta y} \\
w(x, y, t)
\end{array}\right)
$$

where $w(x, y, t)$ represents the displacement along the $z$ axis, and $z_{0}$ the neutral plan ordinate, which distinguishes the compressed and the stretched zones in the plate.

The radius of curvature, $\rho$, due to bending moments caused by contraction of the piezoceramic, can be determined from the resulting stresses and strains in the composite beam (Fig. 7). The plate does not vibrate according to a planar movement but bends. In fact, it is easier to realize bending deformation than planar deformation.

To carry out this modeling, we suppose that we are in the case of small deflections, so that all the lines which are parallel to the neutral line, $r$, have the same radius of curvature [17]:

$$
\frac{d \Phi}{r} \simeq \frac{d \Phi}{x}=\frac{1}{\rho}=\frac{d^{2} w}{d x^{2}},
$$

where $d \phi$ is a small angle between the endpoints of the small beam (Fig. 7). The radius of curvature, $\rho$, due to bending moments caused by the contraction of the piezoceramics, can be determined from the resulting stresses and strains in the composite beam (Fig. 7).

If $z_{0}$ denotes the neutral line ordinate and $z-z_{0}$ the distance between a deformed line and the neutral line, the strain $S_{x x}$ is written:

$$
S_{x x}(z)=\left(z_{0}-z\right) \frac{d^{2} w}{d x^{2}}=\frac{z-z_{0}}{\rho},
$$

where $S_{x x}(z)$ is the strain component along the $x$-axis acting on the plane normal to the $x$-axis.

The resonator is considered to be isotropic, and the stress $T_{i}$ can be expressed by the reduced form as follows:

$$
T_{i}=E_{i} S_{x x}
$$


where $E_{i}$ is the Young modulus of the copper beryllium. As for the piezoelectric part of the plate, the constitutive relationships between the stress, strain, and applied fields can be expressed in terms of the piezoelectric stress relations:

$$
\begin{aligned}
T_{p} & =c^{E} S-e^{t} \mathcal{E}, \\
D & =e S+\epsilon^{S} \mathcal{E},
\end{aligned}
$$

where $\mathcal{E}$ and $D$ are, respectively, the electric field intensity vector and the electric flux density vector. The terms $c^{E}, e$, and $\epsilon^{S}$ are, respectively, the elastic constants short circuit matrix, the voltage coefficients, and the dielectric constants matrixes.

Finally, the stress distributions on the $x$-faces due to an applied electric field in the $z$-direction can be written as follows:

$$
T_{x x}(z)= \begin{cases}E_{i} S_{x x}(z) & h_{p}<z<h_{p}+h_{i} \\ c_{11_{p}}^{E} S_{x x}-d_{31} c_{11_{p}}^{E} \mathcal{E}_{z} & 0<z<h_{p}\end{cases}
$$

where $e^{t}=d_{31} c_{11_{p}}^{E}$.

In order to maintain moment balance, the moment of forces must be zero, as there are no externally applied moments or forces acting on this structure:

$$
M=0=b \int_{z}\left(z-z_{0}\right) T_{x x}(z) d z .
$$

We integrate (22) between zero and $\left(h_{p}+h_{i}\right)$ (Fig. 6), and we use the expressions of $T_{x x}$ given by (21) and (18):

$$
\begin{aligned}
& \int_{0}^{h_{p}}\left(z-z_{0}\right) d_{31} c_{11_{p}}^{E} \mathcal{E}_{z} d z= \\
& \int_{0}^{h_{p}} \frac{\left(z-z_{0}\right)^{2}}{\rho} c_{11}^{E} d z+\int_{h_{p}}^{h_{p}+h_{i}} \frac{\left(z-z_{0}\right)^{2}}{\rho} E_{i} d z .
\end{aligned}
$$

By integrating (23), we deduce that curve $c$ can be expressed as follows:

$$
c=\frac{1}{\rho}=\frac{d^{2} w}{d x^{2}}=\frac{3}{2} \frac{d_{31} \mathcal{E}_{z}}{h_{p} a}
$$

where

$$
\frac{1}{a}=
$$

$\frac{1-2 f_{0}}{1-3 f_{0}+3 f_{0}^{2}+\alpha\left(3 \beta+3 \beta^{2}+\beta^{3}-6 \beta f_{0}-3 \beta^{2} f_{0}+3 \beta f_{0}^{2}\right)}$

and

$$
\alpha=\frac{E_{i}}{c_{11_{p}}^{E}}, \beta=\frac{h_{i}}{h_{p}}, f_{0}=\frac{z o}{h_{p}} .
$$

By taking into account the boundary conditions of a simply supported beam and by integrating (24) twice, we obtain the displacement profile:

$$
w(x)=\frac{3}{4} \frac{d_{31} \mathcal{E}_{z}}{h_{p} a}\left(x^{2}-\frac{\lambda}{2} x\right),
$$

and the static deflection at $\lambda / 4$ is given by

$$
\begin{aligned}
w_{\max }=w\left(\frac{\lambda}{4}\right) & =\frac{-3}{16} \frac{d_{31} \mathcal{E}_{z}}{h_{p}} \frac{(\lambda / 2)^{2}}{a} \\
& =-\frac{3}{16} \frac{d_{31} V_{z}}{h_{p}^{2}} \frac{(\lambda / 2)^{2}}{a},
\end{aligned}
$$

where $V_{z}$ is the voltage applied between the piezoceramic electrodes.

2. Determination of the Dynamic Deflection: In order to increase the deflection, we work at resonance frequency. This explains why, in this subsection, we must determine the dynamic deflection, which is the static deflection times the dynamic amplification factor.

The dynamic amplification factor is the mechanical quality factor $Q_{m}$ of the piezoceramics balanced by the ratio of the strain energy of the entire monomorph $U_{\text {mono }}$ to the strain energy of the piezoelectric layer $U_{\text {piezo }}[18]$ :

$$
Q=Q_{m} \frac{U_{\text {mono }}}{U_{\text {piezo }}}
$$

The stored elastic energy in the piezoelectric layer is given by

$$
U_{\text {piezo }}=\frac{\lambda b}{2} \frac{1}{2} \int_{z} S_{x x} T_{S_{\text {piezo }}} d z .
$$

Substituting into (30) the expressions previously derived for the stress $\left(S_{x x}\right)$ and the strain $\left(T_{s_{\text {piezo }}}\right)$, we obtain

$$
\begin{aligned}
U_{\text {piezo }} & =\frac{\lambda b}{4} \int_{0}^{h_{p}} c_{11_{p}}^{E} S_{x x}^{2} d z \\
& =\frac{\lambda b}{4} \int_{0}^{h_{p}} c_{11_{p}}^{E}\left(\frac{z-z_{0}}{\rho}\right)^{2} d z \\
& =\frac{\lambda b c_{11}^{E} h_{p}^{3}}{12 \rho^{2}}\left(1-\frac{3 z_{0}}{h_{p}}+\frac{z_{0}^{2}}{h_{p}^{2}}\right) \\
& =\frac{3}{16} \lambda b h_{p} d_{31}^{2} \mathcal{E}_{z}^{2} c_{11_{p}}^{E} \frac{1-3 f_{0}+3 f_{0}^{2}}{a^{2}} .
\end{aligned}
$$

In the same way, the stored elastic energy in the entire monomorph is given by

$$
U_{\text {mono }}=\frac{\lambda b}{2} \frac{1}{2} \int_{z} S_{x x}\left(T_{s_{\text {piezo }}}+T_{S_{\text {reson }}}\right) d z .
$$

Substituting into (32) the expressions previously derived for the stress $\left(S_{x x}\right)$ and the strain $\left(T_{s_{\text {piezo }}}+T_{s_{\text {reson }}}\right)$, we obtain

$$
U_{\text {mono }}=\frac{\lambda b}{4}\left(\int_{0}^{h_{p}} c_{11_{p}}^{E} S_{x x}^{2} d z+\int_{h_{p}}^{h_{p}+h_{i}} E_{i} S_{x x}^{2} d z\right)
$$

From (23), we know that

$$
\begin{aligned}
& \int_{0}^{h_{p}} d_{31} c_{11_{p}}^{E} \mathcal{E}_{z}\left(\frac{z-z_{0}}{\rho}\right) d z= \\
& \int_{0}^{h_{p}} c_{11_{p}}^{E}\left(\frac{z-z_{0}}{\rho}\right)^{2} d z+\int_{h_{p}}^{h_{p}+h_{i}} E_{i}\left(\frac{z-z_{0}}{\rho}\right)^{2} d z .
\end{aligned}
$$


Thus, by substituting into (33), we obtain

$$
\begin{aligned}
U_{\text {mono }} & =\frac{\lambda b}{4} \int_{0}^{h_{p}} d_{31} c_{11_{p}}^{E} \mathcal{E}_{z}\left(\frac{z-z_{0}}{\rho}\right) d z \\
& =\frac{\lambda b d_{31} c_{11_{p}}^{E} h_{p}^{2}}{8 \rho}\left(1-\frac{2 z_{0}}{h_{p}}\right) \\
& =\frac{3}{16} \lambda b h_{p} d_{31}^{2} \mathcal{E}_{z}^{2} c_{11_{p}}^{E} \frac{1-2 f_{0}}{a} .
\end{aligned}
$$

Finally,

$$
Q=Q_{m} \frac{a\left(1-2 f_{0}\right)}{1-3 f_{0}+3 f_{0}^{2}}
$$

and

$$
\begin{aligned}
& w_{\mathrm{dyn}}=\left(\frac{\lambda}{4}\right) Q= \\
& Q_{m} \frac{-3}{16} \frac{d_{31} V_{z}}{h_{p}^{2}}\left(\frac{\lambda}{2}\right)^{2}\left(\frac{1-2 f_{0}}{1-3 f_{0}+3 f_{0}^{2}}\right) .
\end{aligned}
$$

Since the dynamic deflection (or the amplitude of vibration) is expressed as a function of geometrical parameters for a given voltage value, it could be feasible to deduce the half-wavelength beam which meets the criteria established in our analysis of the "squeeze film effect."

3. Condition on the Frequency: The resonance frequency, $f_{n}$, is a function of the dimensions of the plate and can be expressed by the following equation:

$$
f_{n}=\left(\frac{\pi}{\lambda / 2}\right)^{2} \sqrt{\frac{G_{b}}{M_{b}}}
$$

in which $M_{b}$ is the total mass per length, expressed as follows:

$$
M_{b}=\rho_{p} h_{p}+\rho_{i} h_{i},
$$

where $G_{b}$ is the flexional rigidity of the monomorph in N.m. $G_{b}$ is the sum of the flexional rigidity of both materials, where $z$ here is measured from the neutral axis:

$$
G_{b}=c_{11_{p}}^{E} b \int_{\text {piezo }} z^{2} d z+E_{i} b \int_{\text {substrate }} z^{2} d z
$$

4. Neutral Line Ordinate: To complement our study, we need to find the neutral line ordinate $z_{0}$ as a function of geometrical parameters. Since the neutral line is situated where stresses cancel each other out and change sign, the sum of the stresses at both sides of the line equals zero. The calculations are carried out under the assumption that the materials are purely elastic, i.e., with a null electric field. At $z_{0}$, we can see that

$$
0=\int_{z} S_{x x}\left(T_{s_{\text {piezo }}}+T_{s_{\text {reson }}}\right) d z
$$

After developing this expression, we find

$$
z_{0}=\frac{h_{p}\left(1+\alpha \beta^{2}+2 \alpha \beta\right)}{2(1+\alpha \beta)} .
$$

TABLE II

List of Parameters.

\begin{tabular}{lr}
\hline \multicolumn{3}{c}{ Mechanical properties of the resonator } \\
\hline Young modulus $E_{i}\left(10^{9} \mathrm{~N} . \mathrm{m}^{-2}\right)$ & 123 \\
Poisson coefficient $\nu_{i}$ & 0.31 \\
\hline \multicolumn{2}{c}{ Mechanical properties of P1-91 ceramic } \\
\hline Piezoelectric constant $e_{31_{p}}\left(\mathrm{C} . \mathrm{m}^{-2}\right)$ & -4.9 \\
Elastic constant & 6.79 \\
$c_{11_{p}}^{E}=\frac{s_{11}^{E}}{\left(s_{11}^{E}\right)^{2}-\left(s_{12}^{E}\right)^{2}}\left(10^{10} \mathrm{~N} . \mathrm{m}^{-2}\right)$ & -247 \\
Charge coefficient & 60 \\
$d_{31}\left(10^{-12} \mathrm{~m} . \mathrm{V}^{-1}\right)$ & \\
Mechanical quality factor & \\
$Q_{m}$ & 1.81 \\
\hline Physical properties of the monomorph structure \\
\hline Elastic constant ratio $\alpha$
\end{tabular}

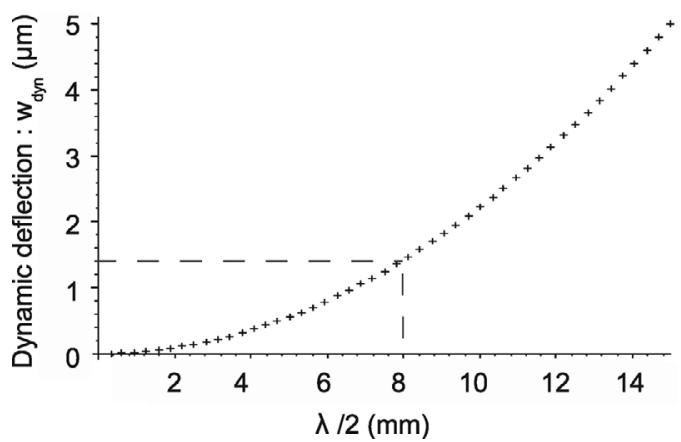

Fig. 8. Dynamic deflection as a function of the half wavelength $(\lambda / 2)$ for a thickness ratio $\beta=2$ and for a supply voltage of $15 \mathrm{~V}$.

5. Results: In this study, we use copper beryllium, which is a passive material, and PZT piezoelectric ceramics, referred to as PI-91, marketed by the Saint-Gobain Quartz Company (Nemours Cedex, France) (the value for $\alpha$ is fixed). The characteristics of the material are indicated in Table II. The voltage supply of the piezoactive ceramics is set to $15 \mathrm{~V}$ in order to avoid dangerous voltages. Moreover, the thickness of the piezoceramic is $1 \mathrm{~mm}$, while the thickness of the substrate is set to $2 \mathrm{~mm}$ (this value has been chosen to guarantee mechanical holding and fabrication considerations), which means that $\beta=2$.

The evolution of the absolute value of the dynamic deflection as a function of the half wavelength is presented in Fig. 8, where the thickness ratio $\beta=2$.

Following the guidelines of the previous section (Fig. 5), it is necessary to design a plate in which the half wavelength is greater than $8.2 \mathrm{~mm}$ (Fig. 8) for a supply voltage of $15 \mathrm{~V}$. The frequency is calculated for $b=49 \mathrm{~mm}$ in order to have a sufficient workspace to meet the ergonomic requirements.

In Fig. 9, the evolution of the resonant frequency obtained from (38), $f_{n}$, is represented as a function of the half wavelength. To meet the condition on the frequency with respect to the squeeze number (Fig. 4), we need 


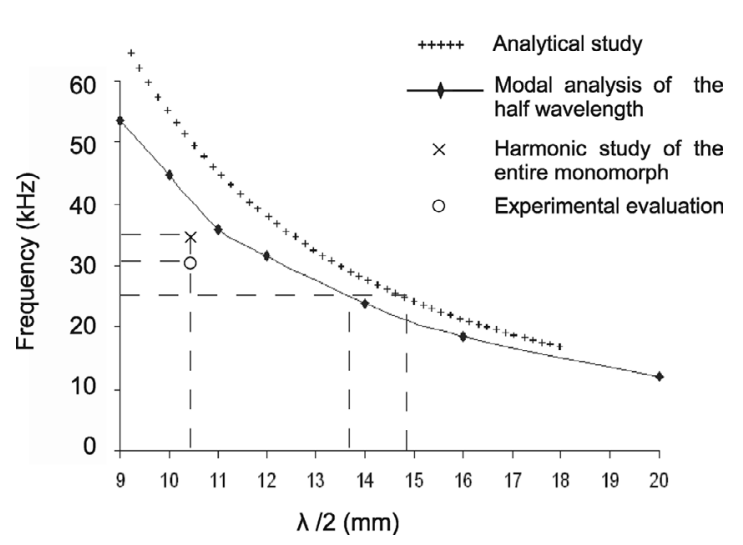

Fig. 9. Natural frequency as a function of the half wavelength: comparison between the analytical and the numerical study.

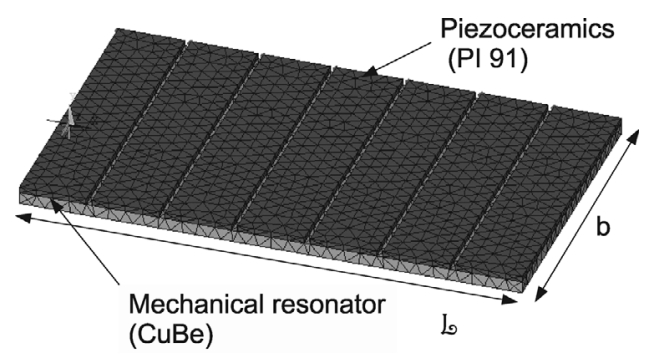

Fig. 10. FE model of the monomorph.

$f_{n}>25 \mathrm{kHz}$, which implies that the half wavelength needs to be less than $14.75 \mathrm{~mm}$.

\section{Numerical Study}

Given that we now know the range of the half wavelength required to change the feeling of roughness under the fingertip for a thickness ratio of $\beta=2$ and supply voltage of $15 \mathrm{~V}$, the final size of the plate remains to be determined. In order to satisfy the ergonomic requirements and to comply with the the delimitations imposed on our analytical study (Fig. 3), a finite element (FE) analysis is performed. The aim of this numerical modeling is to calculate the natural frequencies and the modal shapes of the plate and to perform harmonic analyses.

1. Modal Analysis of Different Half Wavelengths: In this subsection, we calculate the natural frequency of a simply supported beam of length $\lambda / 2$ and of width $b$, as described in the previous section (Fig. 6). This analysis is carried out using the finite element method (FEM) software package ANSYS (ANSYS France SAS, Montigny-leBretonneux, Ile-de-France, France). We consider seven half wavelengths that measure $9 \mathrm{~mm}, 10 \mathrm{~mm}, 11 \mathrm{~mm}, 12 \mathrm{~mm}$, $14 \mathrm{~mm}, 16 \mathrm{~mm}$, and $20 \mathrm{~mm}$. Dimensions and materials used for the simulations are given in Table II and in Appendix B. The piezoceramics are glued onto the mechanical resonator (Fig. 10). Numerical results are obtained for $b=49 \mathrm{~mm}$ in order to allow comparisons with the analytical study.

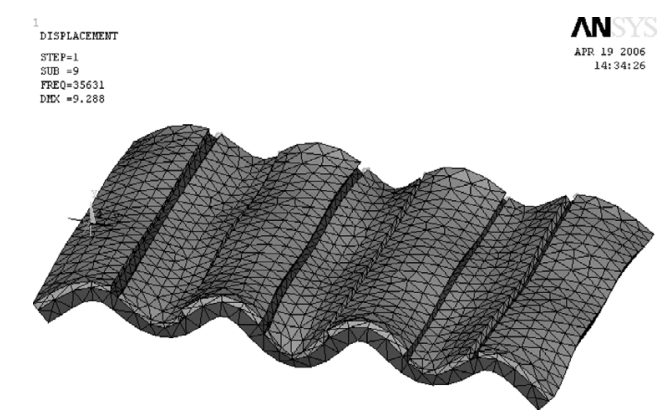

Fig. 11. Result of the modal analysis: deformed shape of the $(8 ; 0)$ mode at $f=35.6 \mathrm{kHz}$ (ANSYS software ${ }^{\circledR}$ ).

The numerical study (Fig. 9) yields a lower boundary for $\lambda / 2(13.6 \mathrm{~mm})$ than the boundary established during the analytical study.

2. Choice of the Final Plate Size-Modal Analysis of the Entire Monomorph: We have at our disposal two types of ceramics; the first type measures $15 \mathrm{~mm}$ and the second type $11 \mathrm{~mm}$. Moreover, the dimension of the piezoceramics has to approach the size of the half wavelength. Since the results of the numerical study also show that the real frequency is probably below the predicted frequency, we choose the $11 \mathrm{~mm}$ ceramic in order to allow for a margin of error. Then, taking into account the requirements for the workspace (Section III-A), we choose a surface length $\mathcal{L}=83 \mathrm{~mm}$ in order to place seven ceramics measuring $11 \mathrm{~mm}$, separated from each other by a distance of $1 \mathrm{~mm}$. The final dimensions of the plate are: $\mathcal{L} \times b=83 \mathrm{~mm} \times 49 \mathrm{~mm}$. This gives us a surface area of $40.67 \mathrm{~cm}^{2}$.

A modal analysis is performed which takes into account the entire monomorph. From this analysis, we find that the $(8 ; 0)$ mode corresponds to a half wavelength of $10.37 \mathrm{~mm}$ in the $x$ direction. The modal shape is presented in Fig. 11 and proves that this resonance frequency $(35.6 \mathrm{kHz})$ is in concordance with our analysis of the squeeze effect $(>25 \mathrm{kHz})$.

3. Harmonic Analysis of the Entire Monomorph: We will now verify that these dimensions meet the amplitude requirements. The FE model of the monomorph is represented in Fig. 10. These ceramics are activated by two electrical signals, with a $180^{\circ}$ phase shift between each. This creates a standing wave.

The harmonic response of the actuator gives the vertical displacement as a function of the frequency (Fig. 12) and shows that the natural frequency is located at $34.77 \mathrm{kHz}$. With this frequency, a vertical displacement of $3.2 \mu \mathrm{m}$ is obtained at the top of the standing wave (cross point reported in Fig. 9).

\section{Experimental Evaluation}

The prototype is presented in Fig. 1. The polarity of each ceramic is chosen in order to ensure that the waves are 


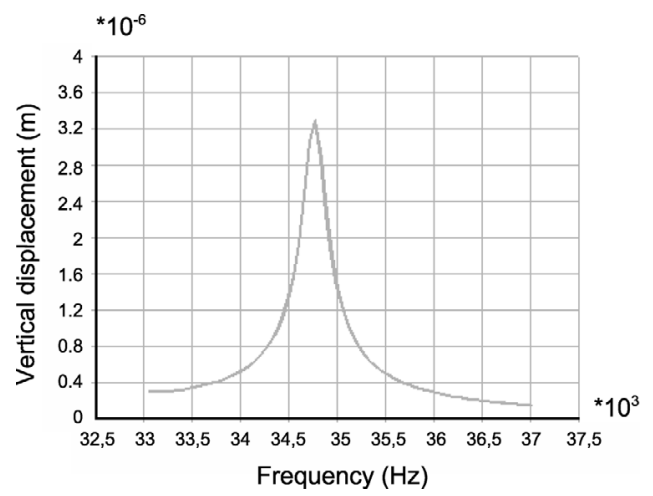

Fig. 12. Results of the harmonic analysis: vertical displacement of a surface point (ANSYS software ${ }^{\circledR}$ ).

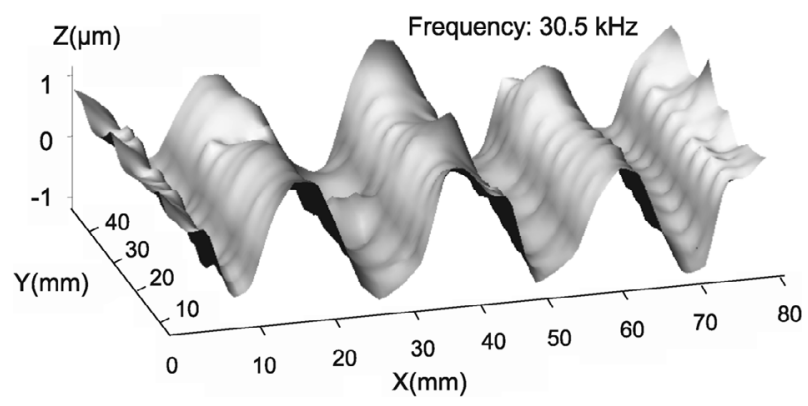

Fig. 13. Laser vibrometric measurements of the $(8 ; 0)$ mode.

produced along the $x$-axis. The device is supplied by one voltage source which is adjusted to a particular mechanical resonance frequency so as to generate the standing wave along the length of the plate. Some experiments with the constructed prototype were carried out in the laboratory to confirm analytical and numerical simulations.

\section{A. Vibration Amplitude Measurement}

The vibration amplitude is measured using a singlepoint LASER Doppler Vibrometer (Model OFV-505, Polytec $\mathrm{GmbH}$, Waldbronn, Germany) linked to a controller (OFV-5000) that is connected to an oscilloscope.

As the results show, a deflection amplitude of about $2.3 \mu \mathrm{m}$ peak to peak is obtained by applying a voltage of $15 \mathrm{~V}$. The resonance frequency is $30.5 \mathrm{kHz}$, (which corresponds to the point marked with a ring in Fig. 9) which gives a squeeze number of 15.9 for $h_{r}=0.6 \mu \mathrm{m}$ (Fig. 13).

The vibration amplitude of the prototype is smaller than the predicted amplitude. This amplitude attenuation may be due to the manufacturing of the monomorph and especially to the gluing phase. More precisely, the gluing phase is crucial for the vibration quality, given our ideal gluing hypothesis. However, according to our analysis of the relative friction, this vibration amplitude of the plate can be sufficient to allow users to perceive a difference. This assumption will be checked in the next section.
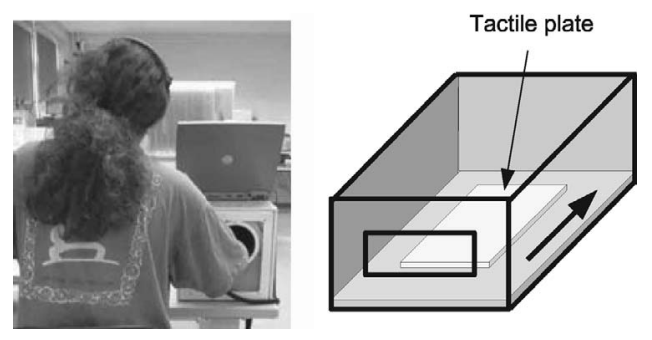

Fig. 14. Experimental setup.

\section{B. Qualitative Study of the Sensation}

In this subsection, we test qualitatively the sensation brought about by the squeeze film phenomenon in our tactile plate. By controlling the amplitude of the ultrasonic vibration, we show that we can vary the tactile sensation. The upper surface of the plate is the touch surface (Fig. 1). The experiment is conducted with twelve naive students aged between 18 and 25. In all conditions, all volunteers (nine men, three women) wear closed headphones (Fig. 14). This allows us to mask the audible cues produced by friction between the finger and the texture. The volunteer's task is to insert his/her hand horizontally into the box and to bring his/her elbow in front of the opening. Then, he or she has to form a straight line with a backward motion of the index finger to discover the surface (the tactile plate) presented in the rectangular opening (Fig. 14). As the feeling of lubrication is obtained by vibrating the surface of the plate, we employ four virtual surfaces which correspond to four (peak to peak) vibration amplitudes: $0 \mu \mathrm{m}, 0.5 \mu \mathrm{m}, 1.2 \mu \mathrm{m}$, and $2.3 \mu \mathrm{m}$. During the first test, the stimuli are presented when the vibration amplitude decreases. Volunteers are asked to say if they feel a difference. If the volunteer answers "yes," he/she is asked to say what has been removed in this phase. All of the subjects feel clearly the difference during the test, and they immediately say that it is "less slippery."

Finally, a second experiment was carried out. Different wave amplitudes were chosen and the volunteers were asked to judge how "slippery" the plate was based on their own personal evaluations. After exploring each "virtual surface," the volunteers rated the slipperiness of the surfaces. The virtual surfaces were chosen randomly.

To control the scale of the numerical differences, the magnitude estimations were multiplied in order to bring them to 100. Results are shown in Table III. In Fig. 15, means for the magnitude estimates are ploted as a function of the vibration amplitudes of the plate. Our results also confirm that the friction coefficient varies as a function of the vibration amplitude.

\section{CONCLUSION}

This work presents theoretical considerations in the design of an ultrasonic tactile plate which are subsequently verified experimentally. Our first step involved analyzing 
TABLE III

Descriptive Statistics.

\begin{tabular}{|c|c|c|c|c|}
\hline & \multicolumn{4}{|c|}{ Perceived slippiness (Magnitude estimate) } \\
\hline & $\begin{array}{c}\text { Ampl. } 1 \\
0 \mu \mathrm{m}\end{array}$ & $\begin{array}{c}\text { Ampl. } 2 \\
0.5 \mu \mathrm{m}\end{array}$ & $\begin{array}{c}\text { Ampl. } 3 \\
1.2 \mu \mathrm{m}\end{array}$ & $\begin{array}{l}\text { Ampl. } 4 \\
2.3 \mu \mathrm{m}\end{array}$ \\
\hline Mean & 12.75 & 19.58 & 56.25 & 81.33 \\
\hline $\begin{array}{l}\text { Maximum } \\
\text { deviation }\end{array}$ & 27.25 & 10.42 & 16.25 & 18.67 \\
\hline $\begin{array}{l}\text { Standard } \\
\text { deviation }\end{array}$ & 12.75 & 8.11 & 8.82 & 10.76 \\
\hline
\end{tabular}

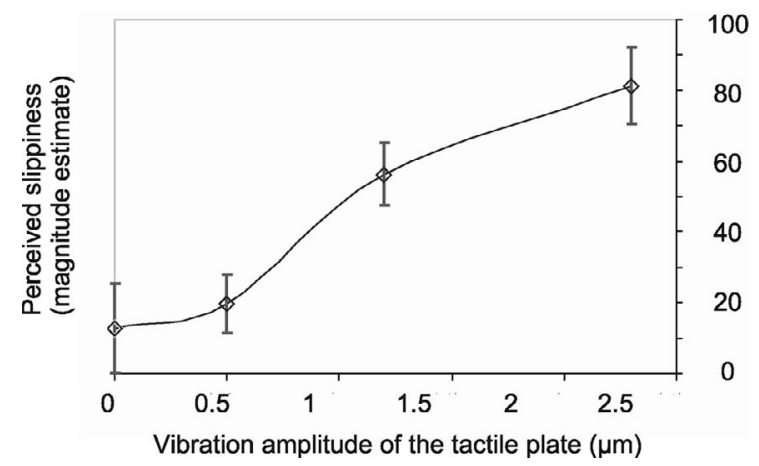

Fig. 15. Mean magnitude estimate as a function of the vibration amplitude of the plate.

the squeeze film effect, which considers the overpressure between a vibrating plate and a fingertip (taking into account the fingers epidermal ridges). This allows us to deduce certain criteria in terms of the vibration amplitude and the average roughness of the plate needed to induce a slippery feeling. In order to generate vibrations in the plate and to meet ergonomic requirements (we need a minimum workspace of $45 \mathrm{~mm} \times 57 \mathrm{~mm}$, with the user's wrist resting on a table), piezoelectric ceramics are glued onto a resonator (substrate) made of copper beryllium. Taking into account the results of our squeeze film analysis, an analytical study is carried out to determine the dynamic deflection of the beam as a function of geometrical parameters. Results allow us to choose the thickness of the plate and the half wavelength required to obtain the vibration amplitude found in the squeeze film study for a given voltage. A numerical study is then carried out to calculate the natural frequency and modal shapes of the plate and to verify the vibration amplitude stipulated in the analytical study.

Experimental trials, which were carried out to check performances of the tactile plate, are presented. The vibration amplitude was measured and the slippery feeling qualitatively checked by means of psycho-physic tests. Moreover, according to this principle, we will be able to simulate different kinds of texture, along the lines of those presented in previous studies, by means of wave modulation.
Appendix A

Resolution of the Differential Equation

$$
\begin{gathered}
\frac{1}{2}[1+\delta \cos (k X)] \frac{d \Psi_{\infty}^{2}}{d X}+\Psi_{\infty}^{2} k \delta \sin (k X)=K \\
\frac{d \Psi_{\infty}^{2}}{\Psi_{\infty}^{2}}=-2 \frac{\delta k \sin (k X)}{1+\delta \cos (k X)} d X \\
\Psi_{1}^{2}=K_{1}(X)[1+\delta \cos (k X)]^{2} \\
K_{1}^{\prime}(X)=\frac{2 K}{[1+\delta \cos (k X)]^{3}} .
\end{gathered}
$$

Since the function $\Psi_{\infty}^{2}$ is a square function, it is necessarily an even function, as

$$
\Psi_{\infty}^{2}(X)=\Psi_{\infty}^{2}(-X)
$$

and

$$
\frac{d \Psi_{\infty}^{2}(X)}{d X}=-\frac{d \Psi_{\infty}^{2}(-X)}{d X}
$$

By replacing $X$ by $-X$ in (43), we obtain

$$
\frac{1}{2}[1+\delta \cos (k X)] \frac{d \Psi_{\infty}^{2}}{d X}+\Psi_{\infty}^{2} k \delta \sin (k X)=-K,
$$

which implies that $K=0$ and that $K_{1}^{\prime}(X)=0$ from (7).

\section{Appendix B}

Density of copper beryllium: $\rho_{i}=8250 \mathrm{~kg} . \mathrm{m}^{-3}$

Material data of the used PZT ceramic PI-91 $11 \times 9 \times 1 \mathrm{~mm}^{3}$ from Saint-Gobain Quartz Company, France.

Density: $\rho_{p}=7410 \mathrm{~kg} . \mathrm{m}^{-3}$

Stiffness matrix $\left[10^{10}\right.$ N.m $\left.{ }^{-2}\right]$ :

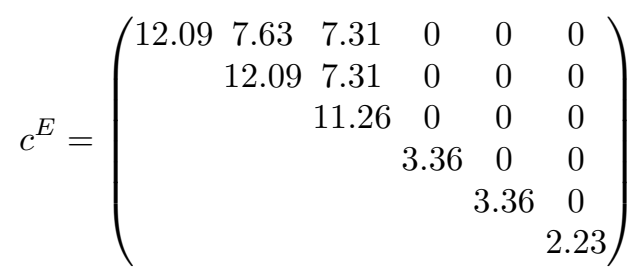

Piezoelectric constants [C.m $\left.{ }^{-2}\right]$ :

$$
e=\left(\begin{array}{cccccc}
0 & 0 & 0 & 0 & 17.1 & 0 \\
0 & 0 & 0 & 0 & 17.1 & 0 \\
-4.9 & -4.9 & 21.4 & 0 & 0 & 0
\end{array}\right)
$$




\section{REFERENCES}

[1] M. Benali-Khoudja, M. Hafez, J.-M. Alexandre, and A. Kheddar, "Tactile interfaces: A state-of-the-art survey," in Proc. 35th Int. Symp. Robotics Automat., Mar. 2004, pp. 721-726.

[2] D. Pawluk, C. P. van Buskirk, J. H. Killebrew, S. Hsiao, and K. Johnson, "Control and pattern specification for high density tactile display," in Proc. ASME Dynam. Syst. Contr. Div., ASME Int. Mech. Eng. Congr. Expo., vol. 64, Oct. 1998, pp. 97-102.

[3] Q. Wang and V. Hayward, "Compact, portable, modular, highperformance, distributed tactile transducer device based on lateral skin deformation," in Proc. 14th Symp. Haptic Interfaces Virtual Environ. Teleoperator Syst., Mar. 2006, pp. 67-72.

[4] M. Takasaki, T. Nara, S. Tachi, and T. Higuchi, "A tactile display using surface acoustic wave," in Proc. 9th IEEE Int. Workshop Robot Human Interact. Commun., RO-MAN 2000, Sep. 2000, pp. 364-367.

[5] M. Takasaki, T. Nara, S. Tachi, and T. Higuchi, "A surface acoustic wave tactile display with friction control," in Proc. 14th IEEE Int. Conf. Microelectromech. Syst., MEMS 2001, Jan. 2001, pp. 240-243.

[6] T. Watanabe and S. Fukui, "A method for controlling tactile sensation of surface roughness using ultrasonic vibration," in IEEE Int. Conf. on Robotics and Automation, 1995, pp. 11341139.

[7] M. Biet, F. Giraud, F. Martinot, and B. Semail, "A piezoelectric tactile display using travelling Lamb wave," in Proc. Eurohaptics, July 2006, pp. 567-570.

[8] T. Maeno, K. Kobayashi, and N. Yamazaki, "Relationship between the structure of human finger tissue and the location of tactile receptors," Bull. JSME Int. J., vol. 41, pp. 94-100, July 1998.

[9] E. O. J. Salbu, "Compressible squeeze films and squeeze bearings," Trans. ASME J. Basic Eng., vol. 86, pp. 355-366, 1964.

[10] M. Wiesendanger, "Squeeze film air bearings using piezoelectric bending elements," Ph.D. dissertation, Ecole Polytechnique Fédérale de Lausanne (EPFL), Switzerland, 2000.

[11] A. M. Smith, G. Gosselin, and B. Houde, "Deployment of fingertip forces in tactile exploration," Exp. Brain Res., vol. 147, no. 2, pp. 209-218, Sep. 2002.

[12] M. Biet, L. Boulon, F. Martinot, F. Giraud, and B. Semail, "Using an ultrasonic transducer: Evidence for an anisotropic deprivation of frictional cues in microtexture perception," in Proc. Joint EuroHaptics Conf. Symp. Haptic Interfaces Virt. Environ. Teleoperator Syst., IEEE World Haptics 2007, Mar. 2007, pp. 385-390.

[13] J. Pasquero and V. Hayward, "Stress: A practical pactile display system with one millimeter spatial resolution and $700 \mathrm{~Hz}$ refresh rate," in Proc. Eurohaptics, 2003, pp. 94-110.

[14] S. J. Lederman and R. L. Klatzky, "Hand movements: A window into haptic object recognition," Cognit. Psychol., vol. 19, pp. 342-368, 1987.

[15] F. Martinot, "Caractérisation du rôle de la dynamique du toucher dans la perception de textures," Ph.D. dissertation, University of Science and Technologies of Lille (USTL), Lille, France, 2006.

[16] L. Landau and E. Lifchitz, Theory of Elasticity, Course of Theoretical Physics. 3rd ed. vol. 7, Oxford, UK: ButterworthHeinemann, 1986.
[17] V. Monturet and B. Nogarede, "Optimal dimensioning of a piezoelectric bimorph actuator," Eur. Phys. J. Appl. Phys., vol. 17, pp. 107-118, 2002.

[18] A D. Nashif, D. I. G. Jones, and J. P. Henderson, Vibration Damping. New York: John Wiley \& Sons, 1985.

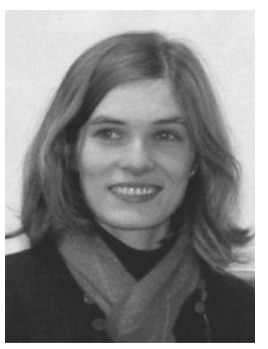

Mélisande Biet was born in France in 1981. She received her B.Sc. degree in electrical engineering from the University of Science and Technologies of Lille, Villeneuve D'Ascq, France, in 2004 and she is currently working toward her Ph.D. degree. Her research interests include mechanical vibrations and modeling and control of piezo-electric actuators.

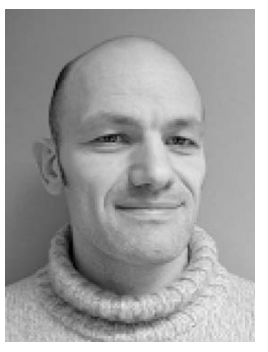

Frédéric Giraud was born in France in 1973. He graduated from the Ecole Normale Supérieure de Cachan, France, in 1996 and received an M.S. degree in electrical engineering in 1997 from the Institut National Polytechnique de Toulouse, France, and a Ph.D. degree from the University of Lille, France, in 2002. He is a member of the electrical engineering and power electronics laboratory of Lille (L2EP), France, as an associate professor. His research deals with the modeling and the control of standing and traveling wave piezo-electric actuators.

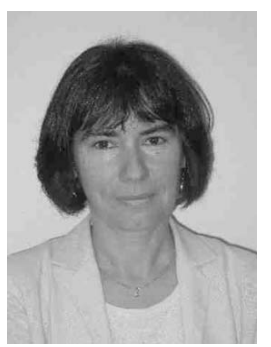

Betty Semail was born France in 1964. She received her Ph.D. degree in 1990 from the University of Paris XI, Orsay, and habilitation degree in 1997 from the University of Science and Technologies of Lille. Since 1990 she has been an associate professor in Ecole Centrale of Lille, and she is now a professor in the University of Lille. She is a member of the Electrical Engineering and Power Electronics Laboratory of Lille (L2EP) and director of a research group focusing on the control of electrical systems. She spent a number of years working on motors; her main field of interest now deals with the modeling and control of piezo-electric actuators, for positioning and force feedback applications. 